\title{
Analysis and implementation of TV-command computer system
}

\author{
Wu Yong Chong, Zhou Yan Hua
}

JiangXi University of Technology

Keywords: TV-command computer system; Implementation;System design; System analysis

\begin{abstract}
TV-command is to control the object to get close to the specified target by TV signal technology. The early TV-command system used artificial recognition and target tracking, whose working process is that when a controlled object is sent out, the TV camera on its head will film the target and its surrounding environment continuously and then send the signal back to the control station. TV receiver of the station displays the image. The operator adjusts the target image to the crosshairs on the screen and sends instructions to the controlled object by radio transmission. The object controls its own movement according to the instructions to get close to the target. In order to improve the comprehensive ability of TV-command computer system, in the article, the TV-command system computer is conducted with reverse principle analysis, and high-speed programmable logic device FPGA and low power processor ARM are applied to implement a new TV-command computer system, which realizes the real-time and high-efficiency information processing, laying a solid foundation for the realization of the system's function and further expansion of functions
\end{abstract}

\section{Introduction}

During the Second World War, Canadians have begun to study TV-command technology. After the war, TV-command technology grew mature gradually, leading to many countries being equipped with TV-command system. With the development of automation technology and rapid change of target recognition technology, based on TV-command technology there appear a lot of new control technologies, such as scene control technology, laser control technique, synthetic aperture radar control technology, satellite positioning control technology, etc. Owing to the unique advantages of TV command---- being accurate and efficient, safe and reliable, along with digital image processing and target recognition technology, it still has its unique research value.

At present, TV-guide technology has become a hot research topic in precise guidance. The main reason is its highly precise guide which can deal with super low altitude targets or low radiation and can be used for broad spectral bands and anti-radio interference etc. But because it requires more about weather condition and cannot be used in rainy and foggy days and the nights, TV-command is developing toward the direction of composite guidance, self guiding, high accuracy and intelligence.

This paper focuses on the analysis of the principle of TV-command computer, and on its basis TV-command computer is designed and fabricated. The new TV-command computer uses ARM9 Chip from Samsung Corp as the core processor to complete the relevant algorithm and the FPGA of Xilinx company to complete the procedure control, which composes the computer system. In the software environment, the article adopts the embedded Linux operating system technology and elaborates bootstrap program, Linux kernel and hardware drivers and the implementation of the relevant functions of the embedded Linux TV- command system. 


\section{The overview of the functions of TV-command computer}

TV-command computer is the key equipment in the target booting system. The computer system has a special detection equipment interface and the state signal recording interface, which can carry out the task of data loading and the total control system parameters processing and loading. It is a task-processing computer with integrated functions in the TV-command booting system.

The computer is a simulation-digital control center with a detection program and parameter calculation mechanism. It receives general control commands and digital flight parameters, completes the calculation of the controlled object and its detection and starting parameter in the starting system and issues the starting control parameter in analog form for the controlled object. The computer has seven functions.

Receive the state information parameter of manual input;

Receive the current information state of the interconnected systems;

Transit and store the general state digital information that is received;

Process the received information according to the established algorithm;

Load information for the controlled object's approach control system before the departure of the object.

Realize the function detection of the object approach control system and the integrated detection of the controller;

Calculate the starting conditions and release the parameters to realize the automation of the starting process.

The automatic detection produces a variety of detection command in order according to an external command and internal state and the time order. It interprets the standard for the analog voltage signal returned by the controlled object and ultimately gives detection results in the form of level signal and communication audio signal.

Through the analysis of the prototype structure and principle of computer system, the structure of the new system that is researched and simulated is shown in Fig .3. The information receiving module receives 16 digit detection codes from the peripheral system and assigns them to the corresponding memory according to the address decoding. After the information receiving ends, they are transmitted to the simulation computer components to be calculated. FPGA can be used for data manipulation and timing control. The computer simulation components need to be digitalized. ARM design is adopted to receive the signal parameters (a total of 9 parameters) stored in FPGA and receiving the manual input parameters at the same time. Process it with the established algorithm and the output it from ARM.

\section{The software design of the computer system}

In the developmental process of embedded Linux, run Linux operating system on the PC machine and use the gcc compiler to compile the application, thus forming the executable file; then through the serial cable or cable, download it on the target board where the ARM Linux can run the program and complete the corresponding task. This process is cross compiled process and the host compiler is a cross compiler.

TV-command peripherals simulation equipment developed by the outsourcing unit can complete automatic detection and single detection of TV-command computer peripheral components and control components equipped general control. Simulation of peripheral equipments can realize the 
self inspection of the system, storage and print of the detection contents and results and the preliminary analysis and location of faults. The peripheral device of TV-command simulation equipment completes the signal detection through IPC, conditioning circuit board, interface board, cooperating with the relevant software.

The connecting relation between the simulation device of TV-command peripheral equipment and peripheral cable is shown in Fig .1.

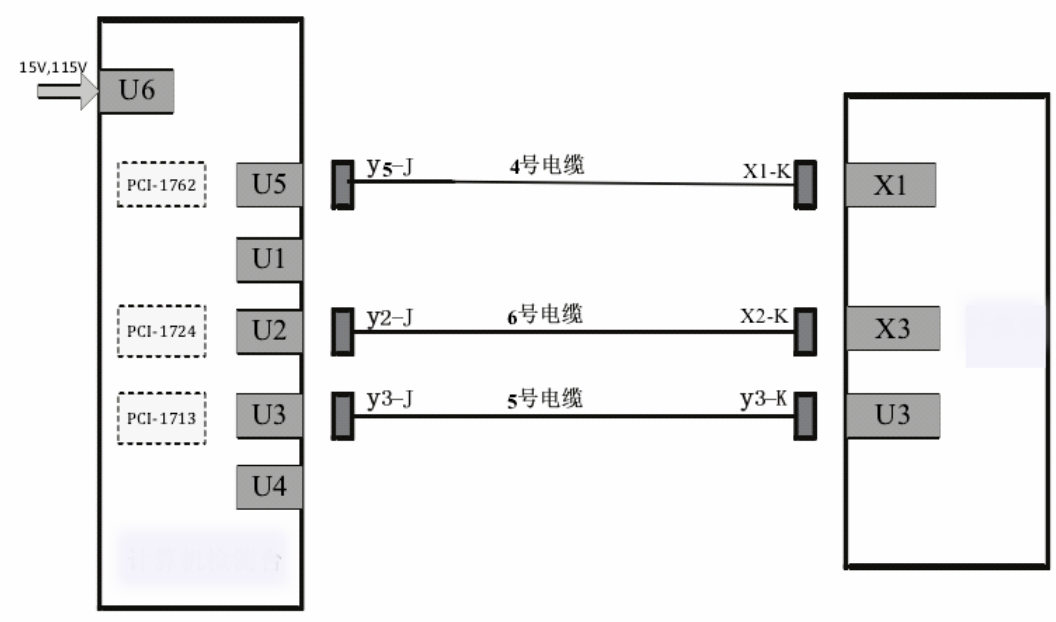

Figure. 1 The schematic diagram of the connecting relation between the simulation device of TV-command peripheral equipment and peripheral cable

According to the temporal relations between signals, we use the interface test special equipment --- VXI Virtual Equipment of TV-command system developed by Beijing Hwa Create Technology Co. Ltd. to collect and analyze data. In reference to the hardware, there are two parts in the interface testing equipment--- data acquisition system, signal conditioning box.

\section{Conclusion}

In this paper, after studying particularly the original TV-command computer assembly structure and working principle, TV-command computer system is designed and simulated by using the modern device. It designs and debugs the hardware circuit of TV command computer system based on ARM and FPGA, establishes the embedded developmental environment, transplants the embedded operating system, compiles the device driving program under the environment of ARM and Linux, designs the established algorithm program of the object of conditions, compile the program in the state of ground detection in FPGA, and designs the environment for self inspection, etc. The main research work is as follows.

Analyze the component structures and principles of the original computer and measure the characteristics and timing sequence of the signals.

Design digital core board and simulation debugging board of the peripheral computer system based on ARM and FPGA.

Complete the schematic diagram and hardware circuit board and debug each module.

Construct embedded Linux environment and the developmental environment for cross compiling.

Transplant ARM and Linux operating system and compile the device driving program under the environment of ARM Linux. 
This paper describes the method of analysis through simulation in the design process. Because the peripheral equipment is objective and unique, it must be careful to analyze the original computer. Besides, the computer system is integrated and sealed in a closed cabin that is protected by inert gas charged in accordance with the aviation standard. It is difficult to open the original computing device to do circuit mapping. Meanwhile, it lacks data of the controlled object, so a lot of work is based on that analysis. Therefore, some signal analysis is not reasonable inevitably, which needs further analysis and improvement.

The analysis of peripheral computer system in the paper is helpful to master the work principle and implement the later maintenance and repair. At the same time, such a simulation method and means can provide some valuable reference for the imitation of other products and can provide some design ideas to guide other technology as well.

\section{References}

[1] Dashevskiy L, Davies T, Nicholson R. Remote control system for electronic devices: U.S. Patent 8,707,372[P]. 2014-4-22.

[2] Yang Y, Liu C, Li C, et al. The recommendation systems for smart TV[C]//Computing, Communication and Networking Technologies (ICCCNT), 2014 International Conference on. IEEE, 2014: 1-6.

[3] Deng J J, Wu D, Liu L. Research on Modeling in CGF System of Air Defense Missile[C]//Applied Mechanics and Materials. 2015, 734: 350-353.

[4] Wu H, Wang J, Zhang X L. User-centered gesture development in TV viewing environment[J]. Multimedia Tools and Applications, 2014: 1-28.

[5] Badi H, Hussein S H, Kareem S A. Feature extraction and ML techniques for static gesture recognition[J]. Neural Computing and Applications, 2014, 25(3-4): 733-741.

[6] Jiao-ling T. SM3 Algorithm Interface Design and Safety Analysis[J]. Information Security and Technology, 2014, 5: 007.

[7] Ergen C W, Dugan M T, Kummer D A, et al. Remote control macro instruction operation: U.S. Patent 8,773,246[P]. 2014-7-8.

[8] Fuhrmann M. Entwicklung einer internetbasierten Evaluationsplattform zur interaktiven Vorlesungsgestaltung[M]. Diplomica Verlag, 2014.

[9] Yang K, Zhao W, Li D, et al. Missile placement analysis based on improved SURF feature matching algorithm[C]//Sixth International Conference on Graphic and Image Processing (ICGIP 2014). International Society for Optics and Photonics, 2015: 94430S-94430S-6.

[10] Ma X X, Li T, Ren Y Q, et al. Research on TV-Command-Guided Air-to-Ground Missile Simulation Training System Based on SIMBox[C]//Advanced Materials Research. 2013, 630: 253-259. 